\title{
ALKALINE PHOSPHATASE IN THE CYTOPLASMIC DROPLET OF MAMMALIAN SPERMATOZOA
}

\author{
K. A. MONIEM AND T. D. GLOVER \\ Unit of Reproductive Biology, University of Liverpool, \\ P.O. Box 147, Liverpool L69 3BX \\ (Received 29th March 1971, accepted 29th Fune 1971)
}

\begin{abstract}
Summary. Alkaline phosphatase activity has been examined in ram, rabbit, hamster and rat spermatozoa. Results have shown that the enzyme is restricted to the cytoplasmic droplet in rabbit and ram spermatozoa but that the intensity of reaction is highest in rabbit spermatozoa. The enzyme could not be demonstrated at all in the spermatozoa of the hamster or rat.

It has also been shown that alkaline phosphatase activity in the droplet of rabbit spermatozoa decreases during epididymal transit and that there is a reduction in activity in degenerating spermatozoa. Degeneration in spermatozoa was produced in rabbits by means of artificial cryptorchidism, and after 2 weeks, activity in the droplets was completely lost.
\end{abstract}

\section{INTRODUCTION}

It is still not clear whether the cytoplasmic droplet of the mammalian spermatozoon is simply a remnant of spermatid cytoplasm that is devoid of any special functional significance, or whether it plays a rôle in the nourishment of the spermatozoon as it passes through the epididymis. Migration of the droplet from the neck of the spermatozoon to the end of its mid-piece coincides with the process of sperm maturation, perhaps the most striking morphological change that this cell undergoes during its passage through the epididymis, and yet the droplet seems to have been overlooked by most workers or disregarded as being functionally insignificant. Its structural details have been described (Bloom \& Nicander, 1961) but recently only the work of Dott \& Dingle (1968) on lysosomal enzymes in bull spermatozoa, and that of Gurya (1963) on cytoplasmic inclusions in the droplet of goat, sheep and buffalo spermatozoa, even hinted at a possible function for the droplet. More recently, Bavdek \& Glover (1970) have reported alkaline phosphatase activity in the droplet of rabbit spermatozoa taken from both intact and ligated epididymides and have further suggested that the droplet in this species might be functionally significant.

However, the incidence and distribution of alkaline phosphatase in the spermatozoa of other mammalian species have not hitherto been clarified, and the present work was undertaken in part as an attempt to fill this gap. It was also felt that, by examining alkaline phosphatase activity in maturing and 
degenerating spermatozoa, some clue to the enzyme's importance might be obtained.

\section{MATERIALS AND METHODS}

Material was obtained for staining from the caput flexure of the epididymis and the cauda epididymidis of four rabbits, six hamsters and six rats immediately after death, and ejaculated spermatozoa were collected from two rabbits by means of an artificial vagina (Walton, 1958). In four rams, samples were obtained from the rete testis, caput flexure and cauda epididymidis as soon as possible after the organs reached the laboratory from the abattoir. Thus, preparation of all epididymal material began within $45 \mathrm{~min}$ of an animal's death and in most of the small animals it was prepared within 10 to $15 \mathrm{~min}$.

In each animal, a small piece of the caput flexure was removed and cut into smaller pieces with fine scissors in a watch glass. The fluid that emerged was then diluted with a small amount of normal saline before proceeding with the preparation. Mature spermatozoa were obtained by incising the cauda epididymidis. The contents at this level welled out of the duct except in the hamster where they are relatively viscous, and in the rat where they are cheesy in consistency. In each case, however, fluid from this area was also diluted with saline, and then a drop was taken and examined microscopically to ensure that the spermatozoa were actively motile. Fluid from the rete testis of rams was collected by means of a fine catheter in a similar way to the method described by Setchell, Scott, Voglmayr \& Waites (1969) for anaesthetized animals. This was done in order to obtain as clean a sample as possible, largely free from contamination with germinal tissue. This was not possible in the rabbit, but it was found that satisfactory samples could be obtained by incising the dorsal pole of the testis at a point just beneath the initial segment of the epididymis.

Every sample was washed three times in normal saline, centrifuging each time at low speed for about $2 \mathrm{~min}$, in order to remove excess epididymal plasma which might itself contain alkaline phosphatase (Bell \& Lake, 1962). The procedure was shown to be absolutely necessary in the golden hamster where the content of alkaline phosphatase in epididymal plasma was so high that, after staining, the spermatozoa were completely obscured on the slides as a result of a generalized reaction.

After the samples were washed, smears were made and these were allowed to dry for a few minutes before being fixed in acetone for about $1 \mathrm{hr}$ at $0^{\circ} \mathrm{C}$. The alkaline phosphatase was demonstrated by the use of a modified form of Gomori's method (Culling, 1963), using sodium $\beta$-glycerylphosphate as substrate at $37^{\circ} \mathrm{C}$ and at a $\mathrm{pH}$ of 9.5 . Controls were incubated in substrate that contained 4 mm-cystine hydrochloride. Different incubation periods were tried but in general, rabbit spermatozoa were incubated at 5, 10 and $20 \mathrm{~min}$, while those of the other species were incubated at $\frac{1}{2}, 1,2,3,4$ and $8 \mathrm{hr}$.

Spermatozoa were taken from the testis and from different levels of the epididymis to determine the effect of sperm maturation on the distribution of alkaline phosphatase. The fate of the enzyme in degenerating spermatozoa was also investigated, using artificially cryptorchid rabbits. The rabbits were made 
cryptorchid by the method previously described by Glover (1959). Spermatozoa were obtained from the epididymis in one animal after 2 days of cryptorchidism, in another after 1 week, and in a third after 2 weeks.

\section{RESULTS}

Alkaline phosphatase activity in the cytoplasmic droplet of rabbit spermatozoa was established and confirmed but the reaction was completely negative in the droplet of spermatozoa from the hamster and rat even after as much as $12 \mathrm{hr}$ incubation. A positive reaction was obtained, however, in the droplets of ram spermatozoa, which reacted rather differently from those of rabbit spermatozoa so the two will be considered separately.

\section{Alkaline phosphatase reaction of rabbit sperm droplets}

Spermatozoa taken from the testis of the rabbit showed a clear positive reaction in the cytoplasmic droplet after only $5 \mathrm{~min}$ incubation. This did not apply to spermatozoa taken from the caput flexure which required $10 \mathrm{~min}$ incubation to produce a comparable effect (Pl. 1, Fig. 1). Spermatozoa from the cauda epididymidis (Pl. 1, Figs 2 and 3 ) and both retained and discarded droplets of ejaculated spermatozoa needed $20 \mathrm{~min}$ incubation to give a good reaction. There was no apparent change in reaction, therefore, when the droplet was discarded in a fresh ejaculate. Activity was always restricted to the cytoplasmic droplet except in mature spermatozoa, in which $4 \mathrm{hr}$ incubation elicited a reaction in the mid-piece also.

\section{Alkaline phosphatase reaction of ram sperm droplets}

A positive reaction was obtained in ram spermatozoa only after $3 \mathrm{hr}$ incubation or longer. In immature spermatozoa, activity was mostly limited to the cytoplasmic droplet, although there was a very faint reaction in the mid-piece (Pl. 1, Fig. 4). In mature spermatozoa, however, using the same period of incubation, the mid-piece reaction was comparatively intense (Pl. 1, Fig. 5). As with rabbit spermatozoa, there was no indication of any reaction in the acrosome.

\section{Alkaline phosphatase reaction in droplets of degenerating spermatozoa}

After 2 days of artificial cryptorchidism in the rabbit, alkaline phosphatase was still clearly detectable in the cytoplasmic droplet, though subjectively it appeared slightly weaker than in control preparations (Pl. 1, Fig. 6). After 7 days, there was a pronounced decrease in the intensity of the reaction (Pl. 1, Figs. 7 and 8) and in samples taken after 14 days of cryptorchidism, it had disappeared altogether except for a very faint reaction in a few droplets (Pl. 1, Fig. 9). It was also noticed that, even after 2 days, the cytoplasmic droplet had ostensibly decreased in size (compare Pl. 1, Figs. 1 and 7).

\section{DISCUSSION}

These results show that the cytoplasmic droplets of spermatozoa of the rabbit and ram are rich in alkaline phosphatase. This is especially true in the rabbit 
where the droplet is rather larger than in ram spermatozoa. The droplets of rat and hamster spermatozoa show no reaction even after a long period of incubation and, although the possible presence of low levels of the enzyme cannot be entirely discounted, its apparent absence is interesting since, in the epididymis of these species, unlike that of rabbit and ram, alkaline phosphatase is also absent from the microvilli (Maneely, 1955; Nicander, 1957; Linnetz \& Amann, 1968; and personal observations).

Wislocki (1950) and Friedlaender \& Fraser (1952) claimed to have noted the occurrence of alkaline phosphatase activity in the head of human and ram spermatozoa, respectively, but this finding was not confirmed in the present work. However, the above authors used no controls and also adopted very long periods of incubation (up to $72 \mathrm{hr}$ ) which could lead to artifact due to nonspecific absorption of the lead content of the stain. There is no record either, in this earlier work, of the spermatozoa having been washed relatively free of plasma. It would seem reasonable to believe, therefore, that, when alkaline phosphatase is present in mammalian spermatozoa, it is mainly concentrated in the cytoplasmic droplet.

Occurrence of activity in the mid-piece is nevertheless of interest, particularly since it appears to increase at this site in association with maturation of the spermatozoa. Moreover, present results indicate a corresponding decrease in activity in the droplet, which suggest that if there is a function involved, it is gradually taken over by the mid-piece. This is perhaps understandable when the droplet is likely to be discarded at ejaculation. The difficulty with histochemical methods of this kind is that some interpretations are bound to be subjective, and Bavdek \& Glover (1970) did not have the impression that the intensity of reaction in the droplet decreased when spermatozoa matured. They did not record the incubation times they used, however, and, in the present work, decreased activity during maturation of the spermatozoa was unmistakable.

There is no doubt, moreover, that severely degenerated spermatozoa are virtually devoid of alkaline phosphatase, although a weak reaction was to be seen in some rabbit spermatozoa after 7 days of artificial cryptorchidism. Even during the first week of artificial cryptorchidism, the cytoplasmic droplet of

\section{EXPLANATION OF PLATE 1}

Preparations were stained by the modified Gomori's method (see text) and all micrographs were taken at the same exposures and at a magnification of $\times 1000$.

Fig. 1. Normal rabbit spermatozoa from caput epididymidis. 10-min incubation.

FIG. 2. Normal rabbit spermatozoa from cauda epididymidis. 10-min incubation.

FIG. 3. Normal rabbit spermatozoa from cauda epididymidis. 20-min incubation.

FIG. 4. Normal ram spermatozoa from rete testis. 4-hr incubation.

Fig. 5. Normal ram spermatozoa from cauda epididymidis. 4-hr incubation.

Frg. 6. Spermatozoa from cauda epididymidis of 2-day artificially cryptorchid rabbit. 20 -min incubation. Note the retained cytoplasmic droplet.

FIG. 7. Spermatozoa from caput epididymidis of 1-week artificially cryptorchid rabbit. 10-min incubation. Note the reduction in size of the cytoplasmic droplet.

Frg. 8. Spermatozoa from cauda epididymidis of 1 -week artificially cryptorchid rabbit. 20 -min incubation.

FIG. 9. Spermatozoa from cauda epididymidis of 2-week artificially cryptorchid rabbit. 20-min incubation. 
PLATE 1
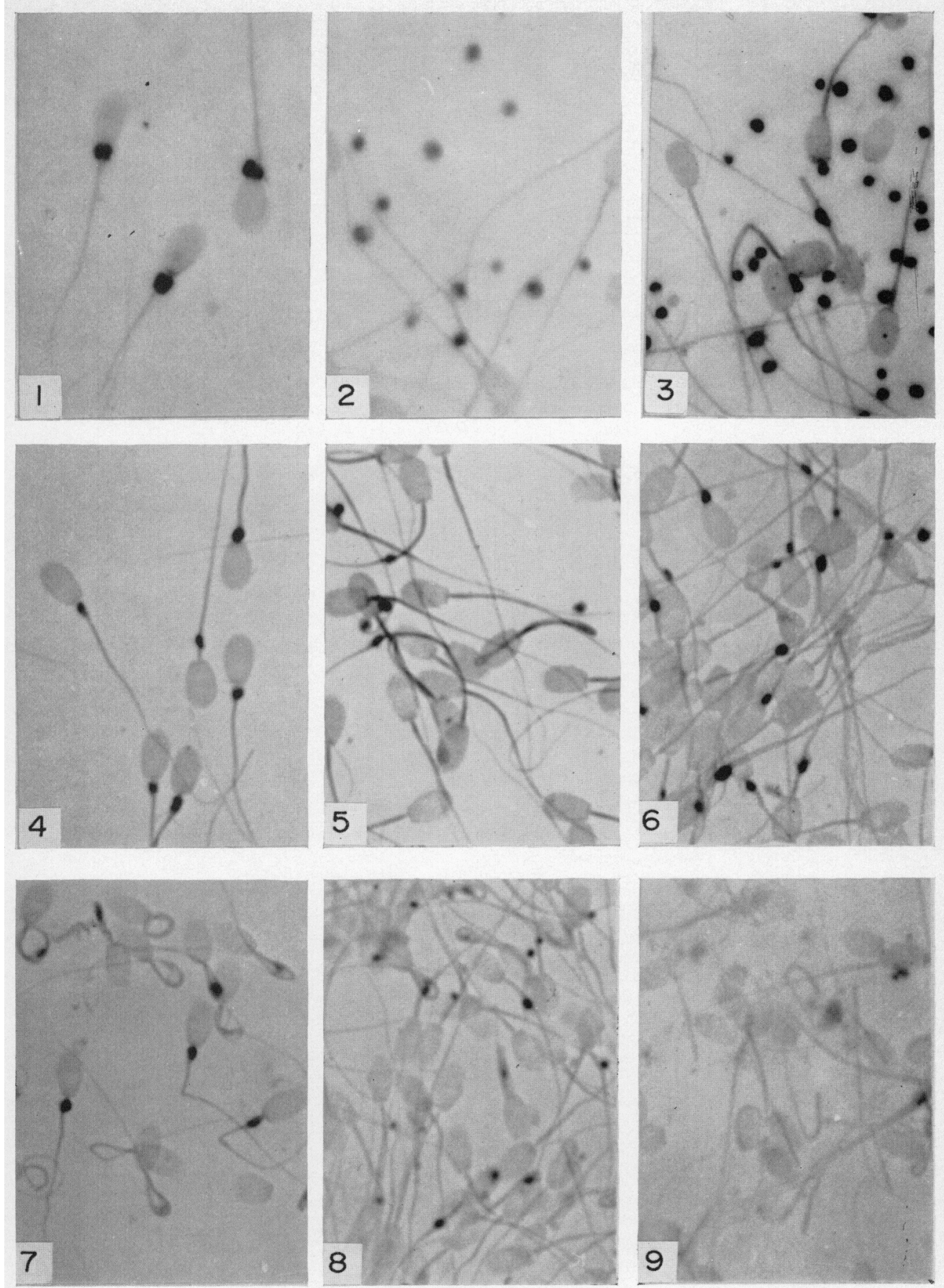

(Facing p. 68) 
rabbit spermatozoa appeared smaller than normal (compare Pl. 1, Figs. 1 and 7) when it was stained for alkaline phosphatase. This effect is difficult to interpret, but illustrates some form of change in the nature of the droplet under these conditions. It is interesting in the light of an earlier finding by Cummins \& Glover (1968) that there is a loss of fertilizing capacity during the first few days of artificial cryptorchidism which corresponds to a high proportion of ejaculated spermatozoa retaining their cytoplasmic droplet. This change in the cytoplasmic droplet may be merely a reflection of more widespread damage to the spermatozoa, but it is suggested from accumulated evidence that more attention might be paid to the droplet itself.

The possibility still cannot be discounted that alkaline phosphatase in spermatozoa, concentrated as it is in the cytoplasmic droplet or in the mid-piece, plays a part in the function of the cell by the dephosphorylation and transport of phosphate groups between epididymal plasma and the sperm cell. If it were true, differences in the intensity of reaction between immature, mature and degenerated spermatozoa suggest that the enzyme is of particular significance in the early maturation of spermatozoa in the epididymis.

\section{AGKNOWLEDGMENTS}

The work was supported by grants from the Ford Foundation and the British Council. Our thanks are also due to the Government of the Sudan for support of one of us (K.A.M.).

\section{REFERENCES}

Bavdek, S. \& Glover, T. D. (1970) Alkaline phosphatase in the cytoplasmic droplet of rabbit spermatozoa. F. Reprod. Fert. 22, 371.

BeLl, D. J. \& LAKE, P. E. (1962) A comparison of phosphomonoesterase activities in seminal plasmas of domestic duck, turkey tom, boar, bull, buck rabbit and of man. F. Reprod. Fert. 3, 363.

BLoom, G. \& Nicander, L. (1961) On ultrastructure and development of the protoplasmic droplet of spermatozoa. Z. Zellforsch. mikrosk. Anat. 55, 833.

Guluing, C. F. A. (1963) Handbook of histopathological techniques, 2nd edn. Butterworth, London.

Gummins, J. M. \& Glover, T. D. (1968) Fertilizing capacity of rabbit spermatozoa following artificial cryptorchidism. F. Endocr. 40, viii.

DotT, H. M. \& Dingle, J. T. (1968) Distribution of lysosomal enzymes in the spermatozoa and cytoplasmic droplet of bull and ram. Expl Cell Res. 52, 523.

Friedlaender, M. \& Fraser, M. (1952) Cytochemical reactions of ram spermatozoa. Expl Cell Res. $3,462$.

Glover, T. D. (1959) Experimental induction of seminal degeneration in rabbits. Stud. Fert. 10, 80.

Guraya, S. S. (1963) Histochemistry of the cytoplasmic droplet in the mammalian spermatozoa. Experientia, 19, 94.

Linnetz, L. J. \& AMANN, R. P. (1968) The male rabbit. II. Histochemistry of the epididymis and ampulla as influenced by sperm output. $\mathcal{F}$. Reprod. Fert. 16, 343.

MANEELy, R. B. (1955) The distribution of polysaccharide complexes and of alkaline glycerophosphatase in the epididymis of the rat. Acta anat. 24, 314.

NICANDER, L. (1957) On the regional histology and cytochemistry of the ductus epididymidis in rabbits. Acta morph. neerl.-scand. 1, 98.

Setchell, B. P., Scott, T. W., Voglmayr, V. K. \& Waites, G. M. H. (1969) Characteristics of testicular spermatozoa and the fluid which transports them to the epididymis. Biol. Reprod. Suppl. 1, 40 .

WaLton, A. (1958) Improvements in the design of an artificial vagina for the rabbit. $\mathcal{F}$. Physiol., Lond. $143,26 \mathrm{P}$.

WisLocli, G. B. (1950) Cytochemical reactions of human spermatozoa and seminal plasma. Anat. Rec. 108,645 . 